\title{
Comparison of DEIM and BPIM to Speed up a POD-based Nonlinear Magnetostatic Model
}

\author{
T. Henneron ${ }^{1}$, L. Montier ${ }^{1,2}$, A. Pierquin ${ }^{1}$ and S. Clénet ${ }^{1}$ \\ ${ }^{1}$ Univ. Lille, Centrale Lille, Arts et Metiers ParisTech, HEI, EA 2697 - L2EP, F-59000 Lille, France \\ ${ }^{2}$ EDF R\&D, THEMIS, 1 Avenue du Général de Gaulle, 92140 Clamart, France
}

\begin{abstract}
Proper Orthogonal Decomposition (POD) has been successfully used to reduce the size of linear Finite Element (FE) problems, and thus the computational time associated with. When considering a nonlinear behavior law of the ferromagnetic materials, the POD is not so efficient due to the high computational cost associated to the nonlinear entries of the full FE model. Then, the POD approach must be combined with an interpolation method to efficiently deal with the nonlinear terms, and thus obtaining an efficient reduced model. An interpolation method consists in computing a small number of nonlinear entries and interpolating the other terms. Different methods have been presented to select the set of nonlinear entries to be calculated. Then, the (Discrete) Empirical Interpolation method ((D)EIM) and the Best Points Interpolation Method (BPIM) have been developed. In this article, we propose to compare two reduced models based on the POD-(D)EIM and on the POD-BPIM in the case of nonlinear magnetostatics coupled with electric equation.
\end{abstract}

Index Terms - Best Points Interpolation Method, Empirical Interpolation Method, Proper Orthogonal Decomposition.

\section{INTRODUCTION}

$\mathrm{T}$ $\mathrm{O}$ reduce the computational time of numerical models depending on the time, model order reduction approaches can be well suited. In the literature, the Proper Orthogonal Decomposition combined with the snapshots approach has been widely presented to solve problems in engineering [1][2]. The POD method consists in performing a projection of the solution of the full FE model onto a reduced basis. Then, the size of the matrix equation to solve can be highly reduced. With a nonlinear behavior law, the POD is not so efficient due to the computation of nonlinear terms. Indeed, at each step of the iterative process which allows solving the problem, the nonlinear entries of the full FE problem must be calculated. The computational cost of this operation penalises the computational time of the POD reduced model. Then, to reduce the calculation cost of nonlinear entries, interpolation methods have been developed. These approaches are based on the computation of a small number of nonlinear entries and on the interpolation of other terms. In the literature, different methods can be found in order to select the set of nonlinear entries. Hence, the (Discrete) Empirical Interpolation method [3][4] and the Best Points Interpolation Method [5][6] have been developed. Both approaches can easily be combined with the POD. In electromagnetic modeling, the POD_(D)EIM was used to study a three phase transformer and an electric machine [7][8], a magneto-quasistatic problem solved by boundary element method including a motion of a subdomain [9] or a nonlinear magnetodynamic problem with a model order reduction of an adaptive subdomain [10].
In this article, we propose to compare the POD_(D)EIM and the POD_BPIM to solve a nonlinear magnetostatic problem coupled with electrical circuit using the vector potential formulation. Firstly, the full model is presented. Secondly, the model order reductions based on the POD, the (D)EIM and the BPIM are described. Finally, the POD_(D)EIM and POD_BPIM are compared with an academic example. The results obtained with the reduced models are also compared in terms of accuracy and computational time using the full model.

\section{Non-Linear Magnetostatic Problem Coupled with ELECTRIC CIRCUITS}

We consider a domain D of boundary $\Gamma\left(\Gamma=\Gamma_{\mathrm{B}} \cup \Gamma_{\mathrm{H}}\right.$ and $\Gamma_{\mathrm{B}} \cap \Gamma_{\mathrm{H}}=0$ ) (Fig. 1). The problem is solved on $\mathrm{D} \times[0, \mathrm{~T}]$ with $\mathrm{T}$ the length of the time interval. The inductors are supposed to be stranded and the eddy current effect is neglected. For the ferromagnetic materials, the nonlinear behaviour law is considered.

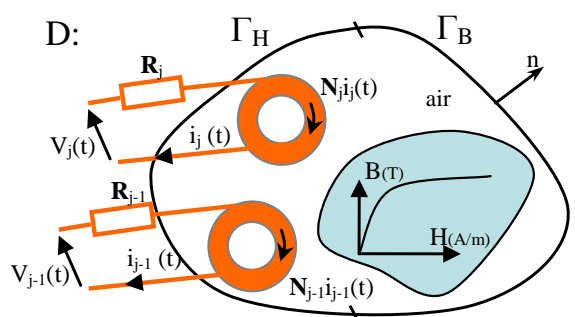

Fig. 1. Non-linear magnetostatic problem coupled with electric circuits

In magnetostatics, the problem is described by the following equations:

$$
\begin{gathered}
\operatorname{curl} \mathbf{H}(\mathbf{x}, \mathrm{t})=\sum_{\mathrm{j}=1}^{\mathrm{N}_{\mathrm{st}}} \mathbf{N}_{\mathrm{j}}(\mathbf{x}) \mathrm{i}_{\mathrm{j}}(\mathrm{t}), \\
\operatorname{div} \mathbf{B}(\mathbf{x}, \mathrm{t})=0,
\end{gathered}
$$

where $\mathbf{x}$ is the position in D, $\mathbf{B}$ is the magnetic flux density, $\mathbf{H}$ is the magnetic field, $\mathbf{N}_{\mathrm{j}}$ and $\mathrm{i}_{\mathrm{j}}$ are the unit current density and the current of the $\mathrm{j}^{\text {th }}$ stranded inductor respectively. $\mathrm{N}_{\mathrm{st}}$ denotes 
the number of stranded inductors. To impose the uniqueness of the solution, boundary conditions must be considered: $\mathbf{B}(\mathbf{x}, \mathbf{t}) \cdot \mathbf{n}=0$ on $\Gamma_{\mathrm{B}}$ and $\mathbf{H}(\mathbf{x}, \mathbf{t}) \times \mathbf{n}=\mathbf{0}$ on $\Gamma_{\mathrm{H}}$ with $\mathbf{n}$ the outward unit normal vector. In order to impose the voltage at the terminals of the stranded inductors, the following relations must be considered:

$$
\frac{d \Phi_{j}(t)}{d t}+R_{j} i_{j}(t)=v_{j}(t) \text { with } j=1, \ldots, N_{s t}
$$

where $R_{j}$ is the resistance, $\Phi_{j}$ is the magnetic linkage flux and $\mathrm{v}_{\mathrm{j}}$ is the voltage of the $\mathrm{j}^{\text {th }}$ stranded inductor. To solve the previous problem, the vector potential formulation can be used. From (2), the vector potential $\mathbf{A}$ is defined such that $\mathbf{B}(\mathbf{x}, \mathrm{t})=\mathbf{c u r l} \mathbf{A}(\mathbf{x}, \mathrm{t})$ with $\mathbf{A}(\mathbf{x}, \mathrm{t}) \times \mathbf{n}=\mathbf{0}$ on $\Gamma_{\text {B }}$. To take into account the nonlinear behavior of the ferromagnetic materials, the magnetic field $\mathbf{H}(\mathbf{x}, \mathbf{t})$ can be expressed by $\mathbf{H}(\mathbf{x}, \mathrm{t})=v_{\mathrm{fp}} \mathbf{B}(\mathbf{x}, \mathrm{t})+\mathbf{H}_{\mathrm{fp}}(\mathbf{B}(\mathbf{x}, \mathrm{t})) \quad$ with $\quad v_{\mathrm{fp}} \quad$ a constant and $\mathbf{H}_{\mathrm{fp}}(\mathbf{B}(\mathbf{x}, \mathrm{t}))=\left(v_{(\mathbf{B})}(\mathbf{x})-v_{\mathrm{fp}}\right) \mathbf{B}(\mathbf{x}, \mathrm{t})$ a virtual magnetization vector depending on the nonlinear reluctivity $\nu_{(\mathbf{B})}(\mathbf{x})$ and on the magnetic flux density. According to (1) and (3), the equations to solve are:

$$
\begin{gathered}
\operatorname{curl}\left(v_{\mathrm{fp}} \operatorname{curl} \mathbf{A}(\mathbf{x}, \mathrm{t})\right)-\sum_{\mathrm{j}=1}^{N_{\mathrm{st}}} \mathbf{N}_{\mathrm{j}}(\mathbf{x}) \mathrm{i}_{\mathrm{j}}(\mathrm{t})=-\operatorname{curl}\left(\mathbf{H}_{\mathrm{fp}}(\operatorname{curl} \mathbf{A}(\mathbf{x}, \mathrm{t}))\right) \\
\frac{\mathrm{d}}{\mathrm{dt}} \int_{\mathrm{D}} \mathbf{A}(\mathbf{x}, \mathrm{t}) \mathbf{N}_{\mathrm{j}}(\mathbf{x}) \mathrm{dD}+\mathrm{R}_{\mathrm{j}} \mathrm{i}_{\mathrm{j}}(\mathrm{t})=\mathrm{v}_{\mathrm{j}}(\mathrm{t}) \text { with } \mathrm{j}=1, \ldots, \mathrm{N}_{\mathrm{st}}
\end{gathered}
$$

The $\mathbf{A}(\mathbf{x}, \mathrm{t})$ field is discretised using edge elements in $3 \mathrm{D}$ and nodal elements in 2D (curl is replaced by grad), while $\mathbf{N}_{\mathrm{j}}(\mathbf{x})$ is computed using facet elements [11]. $\mathrm{A}_{\mathrm{i}}(\mathrm{t})$ denotes the line integral of $\mathbf{A}$ along the $i^{\text {th }}$ edge in $3 \mathrm{D}$ or the value of $\mathbf{A}$ on the $\mathrm{i}^{\text {th }}$ node in 2D. Then, applying the FE method to (4), a system of differential algebraic equations is obtained under the form:

$$
\begin{aligned}
& {\left[\begin{array}{cccc}
\mathbf{M}_{\mathrm{v}_{\mathrm{ff}}} & -\mathbf{F}_{1} & \cdots & -\mathbf{F}_{\mathrm{Nst}} \\
0 & \mathrm{R}_{1} & \cdots & 0 \\
\vdots & & \ddots & 0 \\
0 & \cdots & 0 & \mathrm{R}_{\mathrm{N}_{\mathrm{st}}}
\end{array}\right]\left[\begin{array}{c}
\mathbf{X}_{\mathrm{A}}(\mathrm{t}) \\
\mathrm{i}_{1}(\mathrm{t}) \\
\vdots \\
\mathrm{i}_{\mathrm{Nst}}(\mathrm{t})
\end{array}\right]+\left[\begin{array}{cccc}
0 & \cdots & & 0 \\
\mathbf{F}_{1}^{\mathrm{t}} & \ddots & & \vdots \\
\vdots & & \ddots & \\
\mathbf{F}_{\mathrm{N}_{\mathrm{st}}}^{\mathrm{t}} & 0 & \cdots & 0
\end{array}\right] \frac{\mathrm{d}}{\mathrm{dt}}\left[\begin{array}{c}
\mathbf{X}_{\mathrm{A}}(\mathrm{t}) \\
\mathrm{i}_{1}(\mathrm{t}) \\
\vdots \\
\mathrm{i}_{\mathrm{N}_{\mathrm{st}}}(\mathrm{t})
\end{array}\right]=} \\
& {\left[\begin{array}{c}
0 \\
\mathrm{v}_{1}(\mathrm{t}) \\
\vdots \\
\mathbf{v}_{\mathrm{N}_{\mathrm{s}}}(\mathrm{t})
\end{array}\right]-\left[\begin{array}{c}
\mathbf{M}_{\mathrm{fp}}\left(\mathbf{X}_{\mathrm{A}}(\mathrm{t})\right) \\
0 \\
\vdots \\
0
\end{array}\right]}
\end{aligned}
$$

with $\mathbf{X}_{\mathrm{A}}(\mathrm{t})$ a vector such that $\left(\mathrm{X}_{\mathrm{Ai}}(\mathrm{t})\right)_{1 \leq i \leq \mathrm{Ne}}=\left(\mathrm{A}_{\mathrm{i}}(\mathrm{t})\right)_{1 \leq i \leq \mathrm{Ne}}$ and $\mathrm{N}_{\mathrm{e}}$ the number of Degrees of Freedom. $\mathbf{M}_{v f p}$ is a $\mathrm{N}_{\mathrm{e}} \times \mathrm{N}_{\mathrm{e}}$ square matrix, $\mathbf{F}(\mathrm{t})$ and $\mathbf{M}_{\mathrm{fp}}\left(\mathbf{X}_{\mathrm{A}}(\mathrm{t})\right)$ are $\mathrm{N}_{\mathrm{e}} \times 1$ vectors. Equation (6) can be rewritten in the condensed form:

$$
\begin{aligned}
& \mathbf{M X}(\mathrm{t})+\mathbf{K} \frac{\mathrm{d} \mathbf{X}(\mathrm{t})}{\mathrm{dt}}=\mathbf{F}_{\mathrm{s}}(\mathrm{t})-\mathbf{G}_{\mathrm{fp}}\left(\mathbf{X}_{(\mathrm{t})),}\right. \\
& \mathbf{X}_{(\mathrm{t})}=\left[\begin{array}{c}
\mathbf{\mathbf { X } _ { \mathrm { A } }}(\mathrm{t}) \\
\mathbf{I}(\mathrm{t})
\end{array}\right] \text { and } \mathbf{G}_{\mathrm{fp}}(\mathrm{t})=\left[\begin{array}{c}
\mathbf{M}_{\mathrm{fp}}\left(\mathbf{X}_{\mathrm{A}}(\mathrm{t})\right) \\
\mathbf{0}
\end{array}\right]
\end{aligned}
$$

with $X(t)$ the vector of unknowns of size $\mathrm{N}_{\mathrm{un}}=\mathrm{N}_{\mathrm{e}}+\mathrm{N}_{\mathrm{st}}$ and $\left(\mathrm{I}_{\mathrm{j}}(\mathrm{t})\right)_{1 \leq \mathrm{j} \leq \mathrm{Nst}}=\left(\mathrm{i}_{\mathrm{j}}(\mathrm{t})\right)_{1 \leq \mathrm{j} \leq \mathrm{Nst}}$. To solve $(7)$, an implicit Euler scheme combined with a fixed point approach or the Newton method can be used. In 3D, when $\mathbf{A}$ is not gauged, conjugate gradient methods are used to solve the matrix system. In $2 \mathrm{D}$, due to
Dirichlet boundary conditions, direct solvers can be used.

\section{Model ORdER REDUCTION}

In order to reduce the computational time required to solve the previous problem (7), the POD technique combined with an interpolation method of the nonlinear terms can be applied. The POD and the interpolation method enable to reduce the size of the matrix system and the computational cost of nonlinear terms.

\section{A. Proper Orthogonal Decomposition}

In practice, the number of stranded inductors is very small compared to the size of the vector $\mathbf{X}_{\mathrm{A}}(\mathrm{t})$. Then, the POD is used in order to approximate $\mathbf{X}_{\mathrm{A}}(\mathrm{t})$ in a reduced basis by a vector $\mathbf{X}_{\mathrm{Ar}}(\mathrm{t})$ of size $\mathrm{N}_{\mathrm{X}}\left(\mathrm{N}_{\mathrm{X}}<<\mathrm{N}_{\mathrm{e}}\right)$ such that $\mathbf{X}_{\mathrm{A}}(\mathrm{t})=\Psi_{\mathrm{A}} \mathbf{X}_{\mathrm{Ar}}(\mathrm{t})$. To determine the matrix $\Psi_{\mathrm{A}}$, the Snapshot approach is applied. The full model (7) is solved for $\mathrm{N}_{\mathrm{s}}$ time steps (snapshots). The choice of the snapshots can be based on the first time steps, on the knowledge of the studied device [7][8] or on a greedy algorithm [9]. Then, the snapshot matrix $\mathbf{M}_{\mathrm{s}}$ is defined by $\mathbf{M}_{\mathrm{s}}=\left(\mathbf{X}_{\mathrm{A}}\left(\mathrm{t}_{\mathrm{j}}\right)\right)_{1 \leq \mathrm{j} \leq \mathrm{Ns}}$ with $\mathbf{X}_{\mathrm{A}}\left(\mathrm{t}_{\mathrm{j}}\right)$ the solution at the $\mathrm{j}^{\text {th }}$ time step. Using a singular value decomposition form, the matrix $\mathbf{M}_{\mathrm{s}}$ is equal to $\mathbf{V} \boldsymbol{\sigma} \mathbf{W}^{\mathrm{t}}$ with $\mathbf{V}_{\mathrm{Ne} \times \mathrm{Ne}}$ and $\mathbf{W}_{\mathrm{Ns} \times \mathrm{Ns}}$ orthogonal matrices of singular vectors and $\sigma_{\mathrm{Ne} \times \mathrm{Ns}}$ the diagonal matrix of the singular values. The matrix $\Psi_{\mathrm{A}}$ corresponds to the $\mathrm{N}_{\mathrm{X}}$ first most representative columns of $\mathbf{V}$. To determine $\mathrm{N}_{X}$, we can compare the singular value $\sigma_{\mathrm{j}}$ for $\mathrm{j}=1, \ldots, \mathrm{N}_{\mathrm{s}}$ with an userdefined threshold. In order to preserve the structure of (7), we define a vector $\mathbf{X}_{\mathrm{r}}(\mathrm{t})$ composed of $\mathbf{X}_{\mathrm{Ar}}(\mathrm{t})$ and of $\mathbf{I}(\mathrm{t})$ such that:

$$
\mathbf{X}(\mathrm{t})=\left[\begin{array}{c}
\mathbf{X}_{\mathrm{A}}(\mathrm{t}) \\
\mathbf{I}(\mathrm{t})
\end{array}\right]=\boldsymbol{\Psi} \mathbf{X}_{\mathrm{r}}(\mathrm{t})=\left[\begin{array}{cc}
\boldsymbol{\Psi}_{\mathrm{A}} & \mathbf{0} \\
\mathbf{0} & \mathbf{I}
\end{array}\right]\left[\begin{array}{c}
\mathbf{X}_{\mathrm{Ar}}(\mathrm{t}) \\
\mathbf{I}(\mathrm{t})
\end{array}\right]
$$

with $\Psi$ a block diagonal matrix composed of $\Psi_{\mathrm{A}}$ and of $\mathbf{I d}$, the identity matrix of size $\mathrm{N}_{\mathrm{st}}$. Then, by combining (8) with (7) and by performing a Ritz-Galerkin projection with $\Psi^{\mathrm{t}}$, the reduced model is:

$$
\mathbf{M}_{\mathrm{r}} \mathbf{X}_{\mathrm{r}}(\mathrm{t})+\mathbf{K}_{\mathrm{r}} \frac{\mathrm{d} \mathbf{X}_{\mathrm{r}}(\mathrm{t})}{\mathrm{dt}}=\mathbf{F}_{\mathrm{r}}(\mathrm{t})-\mathbf{G}_{\mathrm{r}}\left(\mathbf{X}_{\mathrm{r}}(\mathrm{t})\right)
$$

with $\quad \mathbf{M}_{\mathrm{r}}=\Psi^{t} \mathbf{M} \Psi, \quad \mathbf{K}_{\mathrm{r}}=\Psi^{t} \mathbf{K} \Psi, \quad \mathbf{F}_{\mathrm{r}}=\Psi^{\prime} \mathbf{F}_{\mathrm{s}} \quad$ and $\mathbf{G}_{\mathrm{r}}\left(\mathbf{X}_{\mathrm{r}}(\mathrm{t})=\Psi^{\mathrm{t}} \mathbf{G}_{\mathrm{fp}}\left(\Psi \mathbf{X}_{\mathrm{r}}(\mathrm{t})\right)\right.$.

\section{B. Interpolation of nonlinear terms}

\section{1) General framework}

To compute the nonlinear entries of $\mathbf{G}_{\mathrm{r}}$, the vector $\mathbf{X}_{\mathrm{Ar}}(\mathrm{t})$ is projected on the original space by $\mathbf{X}_{\mathrm{A}}(\mathrm{t})=\Psi_{\mathrm{A}} \mathbf{X}_{\mathrm{Ar}}(\mathrm{t})$. Then, the entries of $\mathbf{M}_{\mathrm{fp}}\left(\mathbf{X}_{\mathrm{A}}(\mathrm{t})\right)$ can be computed and this vector is projected in the reduced space as $\mathbf{G}_{\mathrm{r}}=\mathbf{\Psi}_{\mathrm{A}}{ }^{\mathrm{t}} \mathbf{M}_{\mathrm{fp}}\left(\mathbf{X}_{\mathrm{A}}(\mathrm{t})\right)$. The computational cost of this operation penalises the computational time of the reduced model. Then, one way to reduce the computational cost of $\mathbf{G}_{\mathrm{r}}$ is to use an interpolation method. In the literature, the BPIM and the (D)EIM have been proposed [3-6]. These approaches are based on the selection of a small number of entries of $\mathbf{M}_{\mathrm{fp}}$ and on the interpolation of the other terms. Firstly, the POD is applied with the snapshots

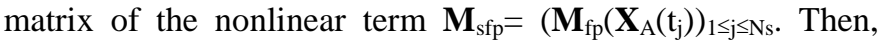
$\mathbf{M}_{\mathrm{fp}}$ is approximated by $\mathbf{M}_{\mathrm{fp}}=\Psi_{\mathrm{fp}} \mathbf{M}_{\mathrm{fp}-\mathrm{r}}$. Secondly, a masked 
projection $\mathbf{P}$, composed of $\mathrm{N}_{\mathrm{x}}$ columns of the identity matrix of size $\mathrm{N}_{\mathrm{e}} \times \mathrm{N}_{\mathrm{e}}$, is performed on $\mathbf{M}_{\mathrm{fp}}$ to select a small number of entries of $\mathbf{M}_{\mathrm{fp}}$ such that $\mathbf{P}^{\mathrm{t}} \mathbf{M}_{\mathrm{fp}}=\mathbf{P}^{\mathrm{t}} \boldsymbol{\Psi}_{\mathrm{fp}} \tilde{\mathbf{M}}_{\mathrm{fp}-\mathrm{r}}$ with $\tilde{\mathbf{M}}_{\mathrm{fp}-\mathrm{r}}$ the approximation of $\mathbf{M}_{\mathrm{fp}-\mathrm{r}}$ due to the interpolation. Then, $\tilde{\mathbf{M}}_{\mathrm{fp}-\mathrm{r}}$ can be computed by:

$$
\tilde{\mathbf{M}}_{\mathrm{fp}-\mathrm{r}}=\left(\mathbf{P}^{\mathrm{t}} \boldsymbol{\Psi}_{\mathrm{fp}}\right)^{-1} \mathbf{P}^{\mathrm{t}} \mathbf{M}_{\mathrm{fp}}=\left(\mathbf{P}^{\mathrm{t}} \boldsymbol{\Psi}_{\mathrm{fp}}\right)^{-1} \mathbf{M}_{\mathrm{fp}-\mathrm{m}}
$$

with $\mathbf{M}_{\mathrm{fp}-\mathrm{m}}$ the vector of selected entries of $\mathbf{M}_{\mathrm{fp}}$ and $\mathbf{P}^{\mathrm{t}} \mathbf{\Psi}_{\mathrm{fp}}$ a square matrix of size $\mathrm{N}_{\mathrm{x}}$. Finally, the vector $\mathbf{M}_{\mathrm{fp}}$ can be interpolated from $\mathbf{M}_{\mathrm{fp}-\mathrm{m}}$ such that $\mathbf{M}_{\mathrm{fp}}=\boldsymbol{\Psi}_{\mathrm{fp}}\left(\mathbf{P}^{\mathbf{t}} \boldsymbol{\Psi}_{\mathrm{fp}}\right)^{-1} \mathbf{M}_{\mathrm{fp}-\mathrm{m}}$. Then, the nonlinear term $\Psi_{\mathrm{A}}{ }^{t} \mathbf{M}_{\mathrm{fp}}\left(\mathbf{X}_{\mathrm{A}}(\mathrm{t})\right)$ of the reduced model (9) is approximated by:

$$
\mathbf{G}_{\mathrm{r}}=\left[\begin{array}{c}
\boldsymbol{\Psi}_{\mathrm{A}}{ }^{\mathrm{t}} \mathbf{M}_{\mathrm{fp}} \\
0
\end{array}\right]=\left[\begin{array}{c}
\boldsymbol{\Psi}_{\mathrm{A}}{ }^{\mathrm{t}} \boldsymbol{\Psi}_{\mathrm{fp}}\left(\mathbf{P}^{\mathrm{t}} \boldsymbol{\Psi}_{\mathrm{fp}}\right)^{-1} \mathbf{M}_{\mathrm{fp}-\mathrm{m}} \\
0
\end{array}\right]
$$

We can note that the matrix $\boldsymbol{\Psi}_{\mathrm{A}}{ }^{\mathrm{t}} \boldsymbol{\Psi}_{\mathrm{fp}}\left(\mathbf{P}^{\mathrm{t}} \boldsymbol{\Psi}_{\mathrm{fp}}\right)^{-1}$ is computed only once. The difference between the BPIM and the (D)EIM is the approach to determine the matrix $\mathbf{P}$.

\section{2) Best points Interpolation Method (BPIM)}

The idea of the BPIM is to minimize the difference between $\mathbf{M}_{\mathrm{fp}-\mathrm{r}}$ and its approximation $\tilde{\mathbf{M}}_{\mathrm{fp}-\mathrm{r}}$ [5][6]. Then, the matrix $\mathbf{P}$ is determined by

$$
\mathbf{P}=\underset{\mathbf{P}}{\arg \min } \sum_{\mathrm{k}=1}^{\mathrm{Ns}}\left(\boldsymbol{\Psi}_{\mathrm{fp}}^{\mathrm{t}} \mathbf{M}_{\mathrm{sp}, \mathrm{k}}-\left(\mathbf{P}^{\mathrm{t}} \boldsymbol{\Psi}_{\mathrm{fp}}\right)^{-1} \mathbf{P}^{\mathrm{t}} \mathbf{M}_{\mathrm{sf}, \mathrm{k}}\right)^{2}
$$

with $\mathrm{M}_{\mathrm{sfp}, \mathrm{k}}$ the $\mathrm{k}^{\text {th }}$ column of the snapshots matrix $\mathbf{M}_{\text {sfp. }}$ The previous equation (12) defines a least squares minimization problem. This kind of problem can be solved by a discrete optimization process based on a Genetic Algorithm (GA). The accuracy of the reduced model depends on the choice of the matrix $\mathbf{P}$. Indeed, the conditioning of the matrix $\mathbf{P}^{\mathbf{t}} \boldsymbol{\Psi}_{\mathrm{fp}}$ depends on $\mathbf{P}$. Then, $\mathbf{P}^{\prime} \Psi_{\mathrm{fp}}$ can be singular or ill-conditioned. The conditioning of $\mathbf{P}^{\mathbf{t}} \Psi_{\mathrm{fp}}$ is added as a constraint to the optimization process.

3) (Discrete) Empirical Interpolation Method (DEIM) With the (D)EIM, the matrix $\mathbf{P}$ is built using a greedy algorithm [3][4]. At each step, the $k+1^{\text {th }}$ column of $\mathbf{P}^{t}$ is defined by the $i_{k+1}$ th column of the identity matrix $\mathbf{I}_{\mathrm{e}}$ of size $\mathrm{N}_{\mathrm{e}}$. The index $i_{k+1}$ is the index for which the error between the vector $\Psi_{\mathrm{fp}, \mathrm{k}+1}$ and its approximation using the k first column of $\mathbf{P}$ is maximum:

$$
\mathbf{P}=\left[\mathbf{P}, \mathbf{I}_{\mathrm{e}}\left(\mathrm{i}_{\mathrm{k}+1}\right)\right] \text { with } \mathrm{i}_{\mathrm{k}+1}=\arg \max \left|\Psi_{\mathrm{fp}, k+1}-\boldsymbol{\Psi}_{\mathrm{fp}}\left(\mathbf{P}^{\mathbf{t}} \boldsymbol{\Psi}_{\mathrm{fp}}\right)^{-1} \mathbf{P}^{\mathbf{t}} \Psi_{\mathrm{f}, k+1}\right|
$$

The matrix $\mathbf{P}^{t} \Psi_{\mathrm{fp}}$ obtained from the (D)EIM is by construction invertible.

\section{APPLICATION}

A 2D magnetostatic example made of a single phase EI transformer at no load supplied at $50 \mathrm{~Hz}$ with a sinusoidal voltage is studied (Fig. 2(a)). The nonlinear behavior of the ferromagnetic core is considered (Fig. 2(b)). The simulation time is composed of three periods with 32 time steps for each period. We compare the currents obtained from two reduced models, POD_(D)EIM and POD_BPIM, with the one obtained using the full model. Fig. 3 presents the evolution of the primary current obtained from the full model for the two first periods of the voltage. In order to construct the reduced models, we consider $\mathrm{N}_{\mathrm{s}}$ equidistributed snapshots extracted to the solving of the full model on the first half period of the voltage. Then, the POD_(D)EIM and POD_BPIM models are solved for all time steps. Fig. 4 present the evolutions of the primary current obtained from both reduced models, and for a different number of snapshots. We can observe that the waveform of the primary current obtained from the POD_BPIM is close to the reference with a low number of snapshots $\left(\mathrm{N}_{\mathrm{s}}=2\right)$. With the POD_(D)EIM, a physical evolution of the current is obtained when $\mathrm{N}_{\mathrm{s}}$ is larger than 12 . To estimate the convergence versus the number of snapshots, an error estimator is defined by

$$
\varepsilon_{\mathrm{MOR}}=\frac{\left\|\mathbf{I}_{\mathrm{FM}}-\mathbf{I}_{\mathrm{MOR}}\right\|_{2}}{\left\|\mathbf{I}_{\mathrm{FM}}\right\|_{2}}
$$

where $\mathbf{I}_{\mathrm{FM}}$ and $\mathbf{I}_{\mathrm{MOR}}$ are the vectors of primary current values at each time step obtained from the full and the one of reduced models. Fig. 5 presents the evolutions of the error versus the number of snapshots. When the number of snapshot is low, the error of the POD_BPIM is weaker than the one from the POD_(D)EIM. When the number of snapshots increases, both reduced models converge toward the same error. The selected entries from the (D)EIM and from the BPIM are presented on the mesh for $\mathrm{N}_{\mathrm{s}}=16$ in Fig. 6 . The localization of the selected entries are different. Nevertheless, the errors of the primary current from both reduced models share the same order of magnitude. In term of local quantity, Fig. 7 presents the distribution of the magnetic flux density computed from the full model for $t=0.01 \mathrm{~s}$. The modulus of the errors between the full model and both reduced models are presented in Fig. 8. The errors from the POD_(D)EIM and the POD_BPIM are located where the direction of $\mathbf{B}$ changes. The maximal values of the error from both reduced models are the same order. In term of computational time, the full and reduced models require 173s and 20s. Then, the speed up is 9 for $\mathrm{N}_{\mathrm{s}}=16$. To compute the POD_(D)EIM and POD_BPIM models, the time is $29 \mathrm{~s}$ and $72 \mathrm{~s}$. These times hold the snapshots computation and the determination of the reduced basis and of the entries of $\mathbf{M}_{\mathrm{fp}}$ to be evaluated. The computational cost with the BPIM is much higher than the one of the (D)EIM, due to the optimization process. The interpolation error introduced by the BPIM depends on the parameters of the optimization process such that the size of population or the number of generations.

\section{CONCLUSION}

The POD combined with the (D)EIM and the BPIM have been developed with a vector potential formulation used to solve a nonlinear magnetostatic problem coupled with electric equations. Two reduced models, based on the POD_(D)EIM and on the POD_BPIM, have been compared on an academic example. With a weak number of snapshots, the error of the POD_BPIM is smaller than the POD_(D)EIM. When the number of snapshots increases, both reduced models converge 
toward the same order of error. Nevertheless, the computational cost of the BPIM is very high compared to the (D)EIM due to the optimization process.

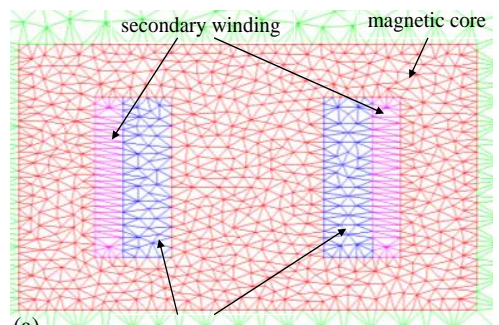

(a) primary winding

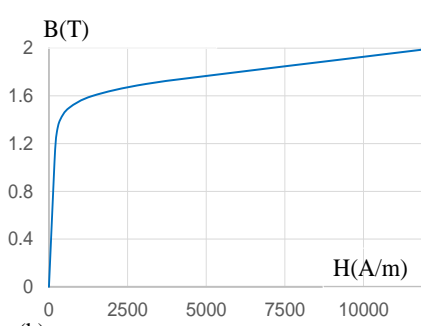

(b)
Fig. 2. Mesh of the geometry (1634 nodes, 2930 elements) (a) and B(H) curve of the ferromagnetic core (b).

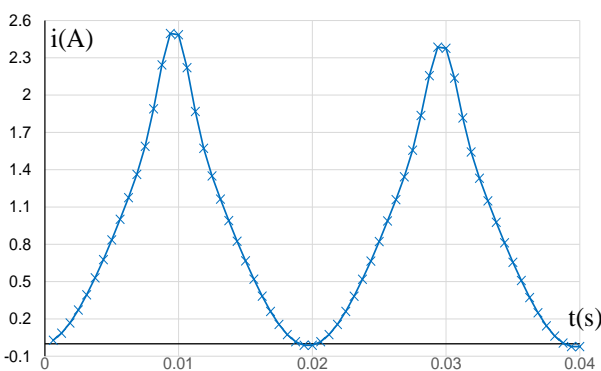

Fig. 3. Evolution of the primary current obtained from the full model.
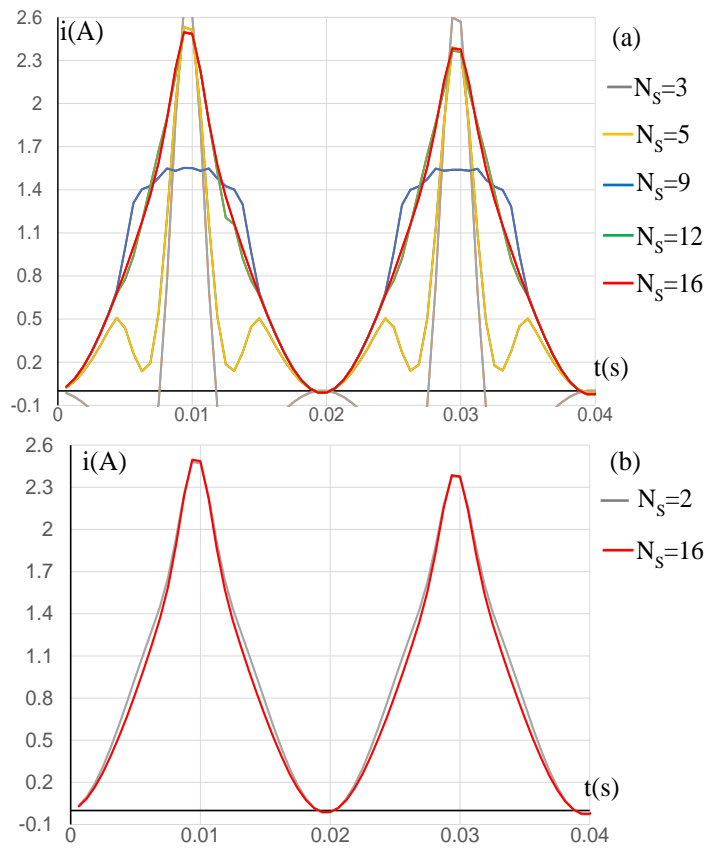

Fig. 4. Evolutions of the primary current obtained from the POD_(D)EIM model (a) and from the POD_BPIM model (b) for different numbers of snapshots.

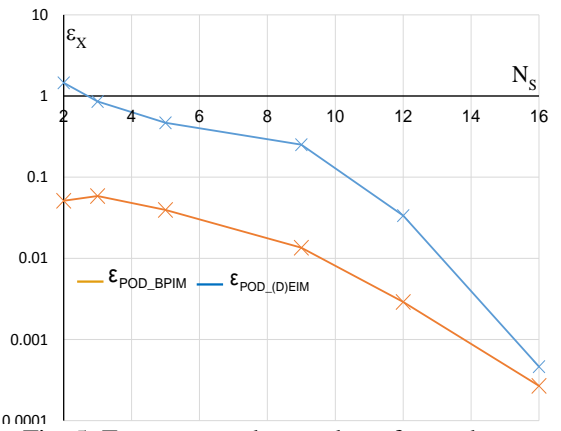

Fig. 5. Error versus the number of snapshots.

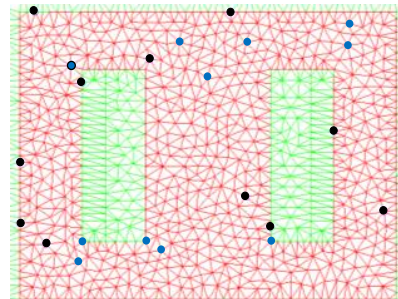

Fig. 6. Selected components by the (D)EIM (black) and the BPIM (blue) with $\mathrm{N}_{\mathrm{S}}=16$.

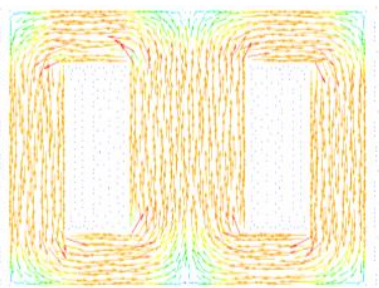

Fig. 7. Distribution of $\mathbf{B}$ from the full model $\left(\mathrm{t}=0.01 \mathrm{~s}, \mathrm{~B}_{\max }=1.68 \mathrm{~T}\right)$.

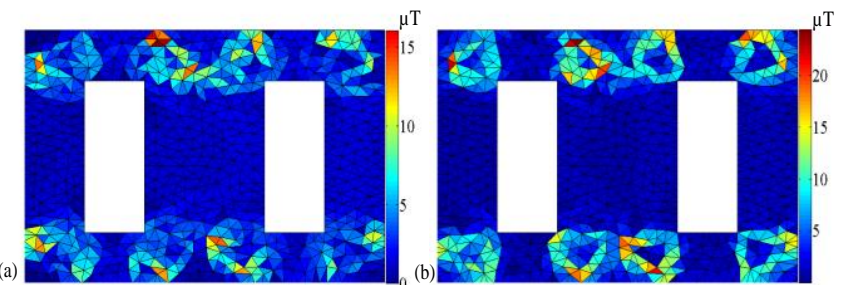

Fig. 8. Error modulus of $\mathbf{B}$ from the POD_(D)EIM (a) and the POD_BPIM (b) $\left(\Delta \mathrm{B}_{\max }=18 \mu \mathrm{T}\right.$ for the POD_(D)EIM and $\Delta \mathrm{B}_{\max }=24 \mu \mathrm{T}$ for the POD_BPIM).

\section{REFER ENCES}

[1] J. Lumley, "The structure of inhomogeneous turbulence", Atmospheric Turbulence and Wave Propagation. A.M. Yaglom and V.I. Tatarski., pp. 221-227, 1967.

[2] L. Sirovich, "Turbulence and the dynamics of coherent structures", $Q$. Appl. Math., vol. XLV, no. 3, pp. 561-590, 1987.

[3] M. Barrault, N. C. Nguyen, Y. Maday, and A. T. Patera. "An "empirical interpolation"method: Application to efficient reduced-basis discretization of partial differential equations", C. R. Acad. Sci. Paris, vol. 339(9), 2004, pp. 667-672, 2004.

[4] S. Chaturantabut and D. C. Sorensen, "Nonlinear Model Reduction via Discrete Empirical Interpolation", SIAM J. Sci. Comput., vol. 32(5), pp. 2737-2764, 2010.

[5] N.C. Nguyen, A.T. Patera and J. Peraire, "A 'best points' interpolation method for efficient approximation of parametrized functions", Numerical Methods in Engineering, vol. 73(4), pp. 521-543, 2008.

[6] D. Galbally, K. Fidkowski, K. Willcox and O. Ghattas, "Non-linear model reduction for uncertainty quantification in large-scale inverse problems", Int. J. Numer. Meth. Engng., vol. 81(12) pp. 1581-1608. doi:10.1002/nme.2746 (2010).

[7] L. Montier, T. Henneron, S. Clénet and B. Goursaud "Transient simulation of an electrical rotating machine achieved through model order reduction", Advanced Modeling and Simulation in Engineering Sciences, Vol. 3, $\mathrm{N}^{\circ}$. 10, 03/2016.

[8] T. Henneron and S. Clénet, "Model-Order Reduction of Multiple-Input Non-Linear Systems Based on POD and DEI Methods", IEEE Trans. Mag., vol. 51(3), 2015.

[9] D. Klis, O. Farle and R. Dyczij-Edlinger, "Model-Order Reduction for the Finite-Element Boundary-Element Simulation of Eddy-Current Problems Including Rigid Body Motion”, IEEE Trans. Mag., 7200404, vol. 52(3), 2016.

[10] Y. Sato, M. Clemens and H. Igarashi, "Adaptive Subdomain Model Order Reduction With Discrete Empirical Interpolation Method for Nonlinear Magneto-Quasi-Static Problems", IEEE Trans. Mag., 1100204, vol. 52(3), 2016.

[11] A. Bossavit, "A rationale for edge-elements in 3-D fields computations", IEEE Trans. Magn.. vol. 24(1), pp 74-79, 1988. 